\title{
Prognostic Factors for Local, Loco-regional and Systemic Recurrence in Early-stage Breast Cancer
}

\author{
Prognosefaktoren für Lokal-, lokoregionäre- und systemische Rezidive \\ beim frühen Mammakarzinom
}

Authors

Affiliation
A. Kümmel, S. Kümmel, J. Barinoff, F. Heitz, J. Holtschmidt, W. Weikel, F. Lorenz-Salehi, A. du Bois, P. Harter, A. Traut, J. U. Blohmer, B. Ataseven

Klinik für Gynäkologie \& Gynäkologische Onkologie, Kliniken Essen-Mitte, Essen

\section{Key words \\ - hormone receptor \\ - HER2/neu \\ - breast cancer \\ Schlüsselwörter \\ - Hormonrezeptor \\ - Her2/neu \\ - Mammakarzinom}

Deutschsprachige Zusatzinformationen online abrufbar unter: www.thieme-connect.de/ ejournals/toc/gebfra

\subsection{4 \\ revised 6.12.2014}

accepted 12.12.2014

Bibliography

DOI http://dx.doi.org/

10.1055/s-0035-1546050

Geburtsh Frauenheilk 2015; 75 :

710-718 @ Georg Thieme

Verlag KG Stuttgart . New York . ISSN 0016-5751

\section{Correspondence}

\section{Dr. Beyhan Ataseven}

Kliniken Essen-Mitte

Klinik für Gynäkologie

\& Gynäkologische Onkologie

Henricistraße 92

45136 Essen

ataseven@gmx.net

\section{Abstract \\ $\nabla$}

Aim: The risk of recurrence in breast cancer depends on factors such as treatment but also on the intrinsic subtype. We analyzed the risk factors for local, loco-regional and systemic recurrence, evaluated the differences and analyzed the risk of recurrence for different molecular subtypes.

Material and Methods: A total of 3054 breast cancer patients who underwent surgery followed by adjuvant treatment at HSK hospital or Essen Mitte Hospital between 1998 and 2011 were analyzed. Based on immunohistochemical parameters, cancers were divided into the following subgroups: luminal A, luminal B (HER2-), luminal B (HER2+), HER2+ and TNBC (triple negative breast cancer).

Results: $67 \%$ of tumors were classified as luminal A, 13\% as luminal B (HER2-), 6\% as luminal B (HER2+), $3 \%$ as HER2+ and $11 \%$ as TNBC. After a median follow-up time of 6.6 years there were 100 local (3.3\%), 32 loco-regional (1\%) and 248 distant recurrences (8\%). Five-year recurrencefree survival for the overall patient collective was $92 \%$. On multivariate analysis, positive nodal status, TNBC subtype and absence of radiation therapy were found to be independent risk factors for all forms of recurrence. Age $<50$ years, tumor size, luminal B (HER2-) subtype and breast-conserving therapy were additional risk factors for local recurrence. Compared to the luminal A subtype, the risk of systemic recurrence was higher for all other subtypes; additional risk factors for systemic recurrence were lymphatic invasion, absence of systemic therapy and mastectomy.

Conclusion: Overall, the risk of local and loco-regional recurrence was low. In addition to nodal status, subgroup classification was found to be an important factor affecting the risk of recurrence.

\section{Zusammenfassung \\ $\nabla$}

Fragestellung: Das Rezidivrisiko beim Mammakarzinom wird durch therapeutische Faktoren, aber auch durch den intrinsischen Subtyp beeinflusst. Diese Arbeit analysiert Risikofaktoren für lokales und lokoregionäres Rezidiv sowie systemische Rezidive, die Evaluation eventuell vorhandener Unterschiede sowie die Analyse des Rezidivrisikos in verschiedenen molekularen Subtypen.

Material/Methoden: Analysiert wurden $3054 \mathrm{~Pa}$ tientinnen mit Mammakarzinom, die an den HSKWiesbaden und Kliniken Essen-Mitte zwischen 1998-2011 operiert und adjuvant behandelt wurden. Anhand immunhistochemischer Parameter erfolgte die Subgruppierung in Luminal A, Luminal B/HER2-, Luminal B/HER2+, HER2+ und TNBC. Ergebnisse: $67 \%$ der Tumoren wurden als Luminal A, 13\% Luminal B/HER2-, 6\% Luminal B/ HER $2+, 3 \%$ HER2+ und 11\% TNBC kategorisiert. Nach einer medianen Nachbeobachtung von 6,6 Jahren traten 100 lokale (3,3\%), 32 lokoregionäre (1\%) Rezidive und 248 Fernmetastasen (8\%) auf. Das metastasenfreie 5-Jahres-Überleben für das Gesamtkollektiv betrug 92\%. In den multivariaten Analysen waren ein positiver Nodalstatus, der TNBC-Subtyp und die nicht durchgeführte Radiotherapie unabhängige Risikofaktoren für alle Rezidivformen. Für das Lokalrezidiv waren zudem Alter $<50$ Jahre, Tumorgröße, Luminal B/HER2- und die brusterhaltende Therapie unabhängige Risikofaktoren. Das Risiko für systemische Rezidive war verglichen mit dem Luminal-A-Subtyp in allen weiteren Subtypen erhöht, neben dem Nachweis einer Lymphgefäßinvasion, nicht erfolgter Systemtherapie und Mastektomie.

Schlussfolgerung: Insgesamt zeigt sich ein niedriges Risiko für lokale und lokoregionäre Rezidive. Neben dem Nodalstatus ist vor allem die Subgruppenklassifikation ausschlaggebend. 


\section{Introduction}

\section{$\nabla$}

With around 75000 new cases every year, breast cancer is by far the most common cancer for women in Germany [1]. As part of national and international certification procedures, German breast centers have been instructed to develop quality indicators for the diagnosis, treatment and follow-up of breast cancer patients. The recommendations of the German Cancer Society (DKG) and the EUSOMA (European Society of Mastology) postulate that the rate for breast-conserving surgery (BCS) should be $70-90 \%$ for $\mathrm{T} 1$ stage tumors [2] and at least $70 \%$ for tumors $<3 \mathrm{~cm}$ [3]. However, there is also the need to comply with another quality criteria, namely, that the maximum local recurrence rate after BCS does not exceed $15 \%$ and the maximum recurrence rate after mastectomy does not exceed $10 \%$ over a period of 10 years [4]. Rates of radical loco-regional surgery have decreased significantly in the last 30 years, starting with the evidence that BCS combined with radiation of the residual breast tissue is at least equal to mastectomy in terms of local recurrence and overall survival [5-9]. Determination of nodal status is required for staging, with nodal status remaining a very important prognostic parameter. However, recently there has also been an important change in the surgical radicality of this staging procedure. Conventional complete axillary lymph node dissection (ALND) which has been established since decades has been replaced by less invasive sentinel lymph node biopsy (SNB) procedures in clinically node-negative patients, after it was shown that SNB is a safe alternative and is associated with significantly lower morbidity [10-13]. Gene expression analysis provides important information about the tumor biology of the breast cancer. Five molecular subtype groups have been identified which provide more subtle information about the risk of recurrence and overall patient survival $[14,15]$. However, for reasons of time and because of the higher costs involved, gene expression analysis is not carried out as a standard procedure in daily clinical routine. Nevertheless, routine determination of specific pathological parameters such as hormone receptor and HER2 status, tumor grade and Ki67 expression can be used for an approximate grouping of the cancer into subtypes which have been found to correlate well with gene expression-based grouping into subtypes $[16,17]$. Several studies have reported a correlation between the classification into subgroups based on pathological investigation and clinical outcomes [18-23]. Several commercially available test methods based on the expression profiles of selected genes are used to determine the therapy of hormone receptor-positive cancers [24] and provide the most individualized treatment plans possible [25].

The aim of this study was to evaluate the data of an unselected patient population from two breast centers (HSK Wiesbaden hospital and Essen-Mitte hospital) accredited by the European Society of Breast Cancer Specialists (EUSOMA) with regard to the prognostic factors for local, loco-regional and systemic recurrence, to identify potential differences and discuss these differences in terms of international benchmarking. The data was also used to examine patterns of recurrence in the different clinical subgroups.

\section{Material and Methods \\ $\nabla$}

Patient population and data acquisition

In this retrospective analysis, the data of patients with a primary diagnosis of early-stage invasive breast cancer obtained from the databases of HSK Wiesbaden and the breast center of Essen-Mitte hospital were evaluated. The observation period covered the years from 1998 to 2011. Follow-up data were collected both from the respective hospitals and from the data submitted annually by gynecologists in private practice linked to the intersectoral breast cancer quality assurance network for Wiesbaden and Essen. Patients with primary systemic recurrence or who underwent neoadjuvant chemotherapy were excluded from the study. Prior to beginning treatment, all patients gave their written consent to invasive procedures as well as to the prospective collection of their data.

\section{Treatment recommendations}

Postoperative systemic and radio-oncologic treatment was administered in accordance with national and international treatment recommendations accepted at the time (e.g. AGO treatment recommendations on breast cancer, S3-guideline on breast cancer, NCCN Guidelines for the treatment of breast cancer, St. Gallen Breast Cancer Conference consensus recommendations [26-29]). It should be noted that anti-HER2 monoclonal antibody therapy with trastuzumab was only available for systemic therapy after it had been approved for general use outside clinical trials in $2006.5 \%$ and $2 \%$ of patients, respectively, rejected the recommendations to undergo chemotherapy or radiation therapy.

\section{Classification into subgroups based on histopathological parameters}

Approximate classification into intrinsic subgroups was done based on pathological parameters, with patients classified into one of the following 5 groups: luminal A: ER and/or PR positive, HER2-, tumor grade $1 / 2$; luminal $B$ (HER2-): ER and/or PR positive, HER2-, tumor grade 3; luminal $B(H E R 2+)$ : ER and/or PR positive, HER2+, tumor grade 1-3; HER2+: ER and PR negative, HER2+, tumor grade 1-3; TNBC (triple negative breast cancer): ER and PR negative, HER2-, tumor grade 1-3.

\section{Definition of study endpoints and statistical analysis}

Incidence rates and associated prognostic parameters were calculated for local recurrence (LR) (defined as recurrence in residual breast tissue or thoracic wall), loco-regional recurrence (RR) (defined as recurrence in the ipsilateral lymph drainage area or axillary/infraclavicular/supraclavicular area) and systemic recurrence (DR) (defined as all other forms of recurrence) along with the corresponding 5-year disease-free survival rates which included local recurrence-free survival (LRFS), loco-regional recurrence-free survival (RRFS) and distant recurrence-free survival (DRFS).

Statistical analysis was done using SPSS Statistics version 20.0. Survival period was defined as the period from the date of diagnosis to the development of recurrence or to the date of the last recorded clinical follow-up. All variables found on univariate analysis $(\mathrm{p}<0.05)$ to affect survival were included in a multivariate model. Hazard ratios and 95\% confidence intervals $(95 \% \mathrm{CI})$ were calculated and validated using the Cox proportional hazards model. Statistical significance was defined as $\mathrm{p}<0.05$ in two-sided test results. 


\section{Results}

\section{Patients and clinical-pathological parameters}

A total of 3054 patients with primary diagnosis of early-stage invasive breast cancer were included in the analysis. Median follow-up was 6.6 years. A total of 380 (12.4\%) recurrences occurred during the follow-up period. Local or loco-regional recurrence occurred in $100(3.3 \%)$ and $32(1 \%)$ of patients, respectively. Distant recurrence was recorded for $8 \%(n=245)$ of the patient population. $9 \%(n=289)$ of patients with primary breast cancer died without signs of recurrence. The 5 -year disease-free survival rate was $88 \%$. The clinical-pathological parameters are listed in 0 Table 1.

On histopathological evaluation, 67\% $(n=2056)$ of tumors were classified as luminal A, $13 \%(n=400)$ as luminal B (HER2-), 6\% $(\mathrm{n}=185)$ as luminal B (HER2+), 3\% $(\mathrm{n}=92)$ as HER2+ and $11 \%$ $(\mathrm{n}=321)$ as TNBC ( 0 Table $\mathbf{1})$.

\section{Local and systemic therapy and impact of anti-HER2 therapy}

Breast-conserving surgery $(B C S)$ was done in $68 \%(n=2081)$ of patients. The re-excision rate was $25 \%(n=762)$. Tumor-free margins were achieved postoperatively in $98 \%$ of cases. Axillary lymph node involvement was investigated in $97 \%$ of all patients (SNB: $33 \%$, ALND: $48 \%$, SNB followed by secondary ALND: 16\%). Local radiation oncology treatment was administered to 2306 (76\%) patients. $94 \%$ of patients had adjuvant systemic therapy (hormone treatment and/or chemotherapy and/or anti-HER2 therapy). 119 of 277 (43\%) patients with HER2-positive cancer received anti-HER2 therapy. Patients who had anti-HER2 therapy had a significantly higher 5-year RRFS (94 vs. $100 \%$; $p=0.011$ ) and distant recurrence-free survival (DRFS) (80 vs. 90\%; $\mathrm{p}=0.011$ ). However, LRFS did not differ significantly between both groups.

\section{Risk factors for local recurrence}

5 -year and 10-year LRFS for the total patient population was $98 \%$ and $94 \%$, respectively ( $\bullet$ Fig. 1 ). 5 -year and 10-year LRFS differed significantly in the intrinsic subgroups: luminal A: 99\%/95\%, luminal B (HER2-): 95\%/90\%, luminal B (HER2+): 97\%/96\%, HER2+: $94 \% / 93 \%$ and TNBC: $94 \% / 91 \%$. On univariate analysis, age $\leq 50$ years, advanced tumor size (T3 and T4), negative hormone receptor status, high tumor grade (G3), positive resection margins and refusal of systemic/radio-oncologic therapy were associated with a significantly higher risk of local recurrence ( $\bigcirc$ Table 2). On multivariate analysis, age $\leq 50$ years, stage T3 tumor, positive node status, the subtypes luminal B (HER2-) and luminal B (HER2+), breast-conserving therapy and refusal of radiation therapy remained as independent negative risk factors (OTable 3).

\section{Risk factors for loco-regional recurrence}

5 -year and 10-year loco-regional recurrence-free survival (RRFS) was $99 \%$ and $98 \%$ respectively (O Fig. 1 ). There were significant differences in 5-year and 10-year RRFS in the intrinsic subgroups: luminal A: 99.5\%/99.1\%, luminal B (HER2-): 99\%/98\%, luminal B (HER2+): 98.5\%/95.1\%, HER2+: 97.5\%/97.5\% and TNBC: $96.2 \% / 96.2 \%$. On univariate analysis, stage T4 tumor, extensive nodal involvement (>10), negative hormone receptor status, type of surgery (mastectomy) and refusal of radiation therapy were associated with a significantly higher risk of recurrence (๑ Table 2). On multivariate analysis, positive node status, TNBC
Table 1 Clinical-pathological parameters and patients characteristics for the total patient population.

\begin{tabular}{|c|c|c|}
\hline Characteristics & $\mathbf{N}$ & $\%$ \\
\hline Total patient population & 3054 & 100 \\
\hline \multicolumn{3}{|l|}{ Age (years) } \\
\hline - median (range) & 61 & 24-95 \\
\hline$>\leq 50$ & 733 & 24 \\
\hline$>>50$ & 2321 & 76 \\
\hline \multicolumn{3}{|l|}{ pTstage } \\
\hline$>\mathrm{T} 1$ & 1819 & 60 \\
\hline$>\mathrm{T} 2$ & 1052 & 34 \\
\hline$>\mathrm{T} 3$ & 120 & 4 \\
\hline$>\mathrm{T} 4$ & 63 & 2 \\
\hline \multicolumn{3}{|l|}{ pN stage } \\
\hline$\rightarrow \mathrm{NO}$ & 2073 & 68 \\
\hline$\rightarrow \mathrm{N} 1$ & 563 & 18 \\
\hline$-\mathrm{N} 2$ & 208 & 7 \\
\hline$\rightarrow \mathrm{N} 3$ & 120 & 4 \\
\hline not determined & 90 & 3 \\
\hline \multicolumn{3}{|l|}{ Hormone receptor status } \\
\hline - $E R \pm P R$ positive & 2641 & 86 \\
\hline ER/PR negative & 413 & 14 \\
\hline \multicolumn{3}{|l|}{ HER2 status } \\
\hline - HER2-negative & 2777 & 91 \\
\hline HER2-positive & 277 & 9 \\
\hline \multicolumn{3}{|l|}{ Tumor grade } \\
\hline G1-2 & 2343 & 77 \\
\hline$\rightarrow \mathrm{G} 3$ & 711 & 23 \\
\hline \multicolumn{3}{|l|}{ Subtypes } \\
\hline - luminal A & 2056 & 67 \\
\hline - luminal B (HER2-) & 400 & 13 \\
\hline - luminal B (HER2+) & 185 & 6 \\
\hline HER2+ & 92 & 3 \\
\hline TNBC & 321 & 11 \\
\hline \multicolumn{3}{|l|}{ Histology } \\
\hline - ductal & 2271 & 74 \\
\hline - lobular & 387 & 13 \\
\hline other & 396 & 13 \\
\hline \multicolumn{3}{|l|}{ Lymphovascular invasion } \\
\hline$>$ no & 2659 & 87 \\
\hline yes & 395 & 13 \\
\hline \multicolumn{3}{|l|}{ Accompanying DCIS } \\
\hline$>$ no & 1576 & 52 \\
\hline$\rightarrow$ yes & 1478 & 48 \\
\hline \multicolumn{3}{|l|}{ Margins } \\
\hline negative & 3005 & 98 \\
\hline positive & 49 & 2 \\
\hline \multicolumn{3}{|l|}{ Multifocal/multicentric } \\
\hline$>$ no & 2498 & 82 \\
\hline yes & 556 & 18 \\
\hline \multicolumn{3}{|l|}{ Type of surgery } \\
\hline breast-conserving & 2081 & 68 \\
\hline - mastectomy & 973 & 32 \\
\hline \multicolumn{3}{|l|}{ Re-excision } \\
\hline$>$ no & 2292 & 75 \\
\hline$\rightarrow$ yes & 762 & 25 \\
\hline \multicolumn{3}{|l|}{ Lymph node resection } \\
\hline$>$ no & 90 & 3 \\
\hline axillary lymph node dissection & 1451 & 48 \\
\hline - Sentinel lymph node biopsy & 1015 & 33 \\
\hline - combined approach & 498 & 16 \\
\hline Systemic therapy & & \\
\hline$>$ no & 170 & 6 \\
\hline yes & 2884 & 94 \\
\hline Radiation therapy of the breast & & \\
\hline$>$ no & 748 & 24 \\
\hline byes & 2306 & 76 \\
\hline
\end{tabular}




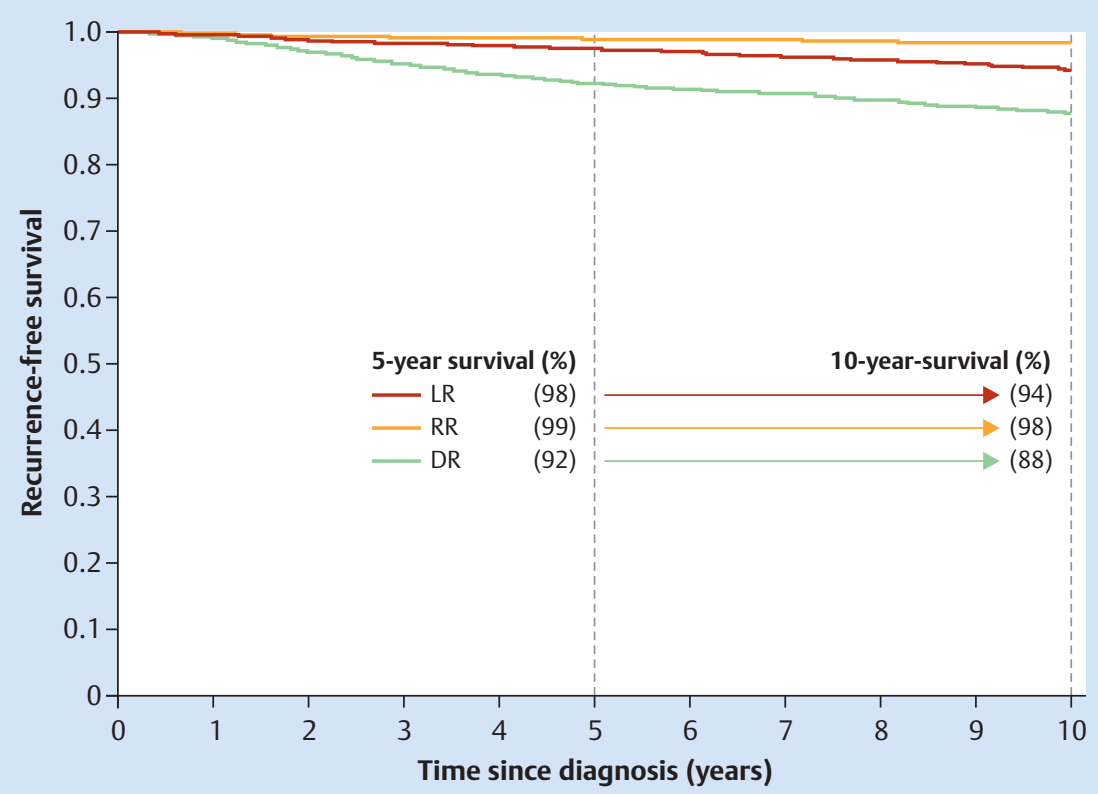

Fig. 1 Kaplan-Meier curve: survival for the total patient population categorized according to the type of recurrence (LR: local recurrence, RR: locoregional recurrence, DR: distant recurrence).

Table 2 Risk factors affecting 5-year and 10-year recurrence-free survival (local, loco-regional and systemic recurrence) on univariate analysis.

\begin{tabular}{|c|c|c|c|c|c|c|}
\hline \multirow[t]{3}{*}{ Characteristics } & \multicolumn{2}{|l|}{ LR } & \multicolumn{2}{|l|}{$\mathbf{R R}$} & \multicolumn{2}{|l|}{ DR } \\
\hline & $n=$ & & $\mathrm{n}=35$ & & $n=2$ & \\
\hline & $\%$ & 5/10-y LRFS & $\%$ & 5/10-y RRFS & $\%$ & 5/10-y DRFS \\
\hline Age (years) & & $p<0.05$ & & n.s. & & n.s. \\
\hline$>\leq 50$ & 40 & 96/91 & 28 & 99/98 & 29 & $91 / 86$ \\
\hline$>>50$ & 60 & $98 / 96$ & 72 & $99 / 98$ & 71 & $93 / 89$ \\
\hline pTstage & & $p<0.05$ & & $p<0.05$ & & $p<0.05$ \\
\hline - $\mathrm{T} 1 \mathrm{a} / \mathrm{mic}$ & 8 & $96 / 91$ & 6 & 99/99 & 3 & $97 / 88$ \\
\hline - $\mathrm{T} 1 \mathrm{~b}$ & 19 & $98 / 92$ & 9 & 99/99 & 7 & $97 / 94$ \\
\hline$\rightarrow \mathrm{T} 1 \mathrm{c}$ & 35 & $98 / 95$ & 41 & $99 / 98$ & 26 & $95 / 91$ \\
\hline$>\mathrm{T} 2$ & 30 & $98 / 96$ & 28 & $99 / 98$ & 47 & $88 / 84$ \\
\hline - $\mathrm{T} 3$ & 7 & 93/93 & 6 & $98 / 98$ & 8 & $83 / 71$ \\
\hline - $\mathrm{T} 4$ & 1 & $96 / 96$ & 9 & $93 / 93$ & 9 & $59 / 52$ \\
\hline pN stage & & n.s. & & $p<0.05$ & & $p<0.05$ \\
\hline - NO & 62 & $98 / 95$ & 38 & 99/99 & 41 & $96 / 93$ \\
\hline - N1 & 24 & $97 / 91$ & 31 & $98 / 97$ & 24 & $90 / 80$ \\
\hline - N2 & 8 & $97 / 96$ & 12 & $97 / 97$ & 18 & $77 / 72$ \\
\hline - N3 & 3 & $97 / 97$ & 16 & $94 / 94$ & 16 & $66 / 56$ \\
\hline - not determined & 3 & $92 / 91$ & 3.1 & $98 / 98$ & 0.8 & $98 / 97$ \\
\hline Hormone receptor status & & $p<0.05$ & & $p<0.05$ & & $p<0.05$ \\
\hline - ER $\pm P R$ positive & 77 & $98 / 95$ & 63 & 99/99 & 75 & $94 / 89$ \\
\hline - ER/PR negative & 23 & $94 / 92$ & 38 & $96 / 96$ & 25 & $85 / 81$ \\
\hline HER2 status & & n.s. & & n.s. & & $p<0.05$ \\
\hline - HER2-negative & 90 & $98 / 94$ & 84 & 99/98 & 84 & $93 / 89$ \\
\hline - HER2-positive & 10 & $97 / 95$ & 16 & 98/96 & 16 & $86 / 79$ \\
\hline Tumor grade & & $p<0.05$ & & n.s. & & $p<0.05$ \\
\hline > $\mathrm{G} 1-2$ & 66 & $98 / 95$ & 69 & $99 / 98$ & 58 & $95 / 91$ \\
\hline - G3 & 34 & $95 / 92$ & 31 & $98 / 98$ & 42 & $86 / 79$ \\
\hline Subtypes & & $p<0.05$ & & $p<0.05$ & & $p<0.05$ \\
\hline - luminal $\mathrm{A}$ & 50 & 99/95 & 41 & 99/99 & 44 & $95 / 92$ \\
\hline - luminal B (HER2-) & 21 & $95 / 90$ & 13 & $99 / 98$ & 22 & $86 / 78$ \\
\hline - luminal B (HER2+) & 6 & $97 / 96$ & 9 & 99/95 & 9 & $89 / 81$ \\
\hline > HER2+ & 4 & $94 / 93$ & 6 & $98 / 98$ & 7 & $80 / 75$ \\
\hline - TNBC & 19 & $94 / 91$ & 31 & $96 / 96$ & 17 & $86 / 83$ \\
\hline Histology & & n.s. & & $p>0.05$ & & $p>0.05$ \\
\hline - ductal & 81 & $98 / 92$ & 78 & $99 / 98$ & 74 & $92 / 88$ \\
\hline - lobular & 5 & $98 / 98$ & 6 & 99/99 & 12 & $92 / 88$ \\
\hline - other & 14 & $97 / 95$ & 17 & $99 / 99$ & 14 & $92 / 87$ \\
\hline
\end{tabular}




\begin{tabular}{|c|c|c|c|c|c|c|}
\hline \multirow[t]{3}{*}{ Characteristics } & \multicolumn{2}{|l|}{ LR } & \multicolumn{2}{|l|}{ RR } & \multicolumn{2}{|l|}{ DR } \\
\hline & $n=100$ & & $n=35$ & & $n=2$ & \\
\hline & $\%$ & 5/10-y LRFS & $\%$ & 5/10-y RRFS & $\%$ & 5/10-y DRFS \\
\hline Lymphovascular invasion & & n.s. & & n.s. & & $p<0.05$ \\
\hline$>$ no & 87 & $98 / 95$ & 84 & 99/99 & 75 & $94 / 89$ \\
\hline D yes & 13 & $98 / 95$ & 16 & 99/98 & 25 & $83 / 77$ \\
\hline Accompanying DCIS & & n.s. & & n.s. & & n.s. \\
\hline$>$ no & $49(49)$ & $98 / 95$ & $15(47)$ & $99 / 99$ & 60 & $92 / 88$ \\
\hline D yes & $51(51)$ & $97 / 94$ & $17(53)$ & $99 / 98$ & 40 & $93 / 88$ \\
\hline Margins & & $p<0.05$ & & n.s. & & n.s. \\
\hline negative & 95 & $98 / 95$ & 100 & $99 / 98$ & 98 & $93 / 88$ \\
\hline positive & 5 & $93 / 88$ & 0 & $100 / 100$ & 2 & $90 / 90$ \\
\hline Multifocal/multicentric & & n.s. & & n.s. & & $p<0.05$ \\
\hline no & 83 & $98 / 95$ & 81 & 99/98 & 75 & $93 / 89$ \\
\hline D yes & 17 & $97 / 92$ & 19 & 99/99 & 25 & $89 / 80$ \\
\hline Type of surgery & & n.s. & & $p<0.05$ & & $p<0.05$ \\
\hline breast-conserving & 70 & $98 / 95$ & 47 & $99 / 99$ & 47 & $95 / 91$ \\
\hline mastectomy & 30 & $97 / 90$ & 53 & $98 / 97$ & 53 & $86 / 79$ \\
\hline Re-excision & & n.s. & & n.s. & & $p<0.05$ \\
\hline$>$ no & 69 & $98 / 95$ & 75 & 99/98 & 81 & $92 / 87$ \\
\hline - yes & 31 & $96 / 94$ & 25 & $99 / 98$ & 19 & $94 / 90$ \\
\hline Lymph node dissection & & n.s. & & n.s. & & $p<0.05$ \\
\hline none & 3 & $98 / 92$ & 3.1 & 99/99 & 0.8 & $97 / 97$ \\
\hline - ALND & 65 & $97 / 94$ & 78 & $99 / 98$ & 78 & $89 / 84$ \\
\hline$>\mathrm{SNB}$ & 18 & $98 / 96$ & 12.5 & $99 / 99$ & 10.2 & $97 / 90$ \\
\hline SNB + ALND & 14 & $97 / 95$ & 6.2 & $99 / 99$ & 11 & $94 / 92$ \\
\hline Systemic therapy & & n.s. & & n.s. & & $p<0.05$ \\
\hline no & 13 & $93 / 88$ & 13 & $97 / 97$ & 11 & $85 / 79$ \\
\hline yes & 87 & $98 / 95$ & 87 & 99/99 & 89 & $93 / 88$ \\
\hline Radiation therapy & & $p<0.05$ & & $p<0.05$ & & $p<0.05$ \\
\hline$>$ no & 38 & $96 / 90$ & 56 & $97 / 96$ & 31 & $90 / 83$ \\
\hline yes & 62 & $98 / 96$ & 44 & $99 / 99$ & 69 & $93 / 89$ \\
\hline
\end{tabular}

LR: local recurrence; RR: loco-regional recurrence; DR: distant recurrence; LRFS: local recurrence-free survival; RRFS: loco-regional recurrence-free survival; DRFS: distant recurrence-free survival; y: year; DCIS: ductal carcinoma in situ; SNB: sentinel lymph node biopsy; ALND: axillary lymph node dissection; n. s.: not significant

subtype and refusal of radiation therapy remained independent negative prognostic factors ( Table 3 ).

\section{Risk factors for systemic recurrence}

5-year and 10-year distant recurrence-free survival (DRFS) for this patient population was $92 \%$ and $88 \%$ respectively ( $\bullet$ Fig. 1 ). However there were significant differences in 5-year and 10-year DRFS in the intrinsic subgroups: luminal A: 95\%/92\%, luminal B (HER2-): 86\%/78\%, luminal B (HER2+): 89\%/81\%, HER2+: $80 \% /$ $75 \%$ and TNBC: $86 \% / 83 \%$ ( Fig. 2). On univariate analysis stageT3/4 tumor, extensive nodal involvement (N2/3), negative hormone receptor status, positive HER2 status, high tumor grade, lymph node invasion, multifocality/multicentricity, type of surgery (mastectomy, as well as axillary lymph node dissection) and refusal of systemic and radio-oncologic therapy were found to be associated with a significantly higher risk of recurrence ( Table 2). On multivariate analysis, tumor size (> T1), node positivity, all subtypes (compared with reference type luminal A), evidence of lymph node involvement, type of surgery (mastectomy) and refusal of systemic and radiation therapy were independent negative risk factors ( Table 3 ).

\section{Comparison of recurrence risk between} the intrinsic subgroups

On multivariate analysis, a significant increase of risk of local recurrence (LR) was found for the subgroups luminal B (HER2-) (HR: 2.4; 95\% CI: 1.4-4.1; $\mathrm{p}=0.001$ ) and TNBC (HR: 2.4; 95\% CI: $1.4-4.2 ; \mathrm{p}=0.001$ ) compared to the luminal A tumor subgroup which served as reference ( $\bullet$ Table 3 ). For loco-regional recurrence (RR) the difference was only significant for the TNBC group (HR: 5.4; 95\% CI: 2.7-11; p=0.001). All non-luminal A tumors were associated with a significantly higher risk of systemic recurrence (DR): luminal B (HER2-) (HR: 2.0; 95\% CI: 1.4-2.8; $\mathrm{p}<0.001$ ), luminal B (HER2+) (HR: 1.9; 95\% CI: 1.2-3.0; $\mathrm{p}=0.007), \mathrm{HER} 2+(\mathrm{HR}: 2.4 ; 95 \% \mathrm{CI}: 1.4-4.1 ; \mathrm{p}=0.001)$ and TNBC (HR: $2.2 ; 95 \%$ CI 1.5-3.2; $\mathrm{p}=0.001$ ).

\section{Comparison of risk factors LR, RR and DR}

On multivariate analysis ( $\bullet$ Table $\mathbf{3}$ ) the following parameters were found to be associated with an increased risk for all types of recurrence (LR, RR, DR): nodal positivity, TNBC subgroup and refusal of radio-oncologic therapy. Status of the final margins and multifocality/multicentricity were not associated with increased risk. Age $\leq 50$ was a prognostic factor for LR but not for RR or DR. Tumor size had a negative effect on LR and DR but was not found to affect RR. Signs of tumor invasion of the lymphatic vessels were associated with increased DR but not with increased LR or RR. Patients who had undergone mastectomy had a low risk 


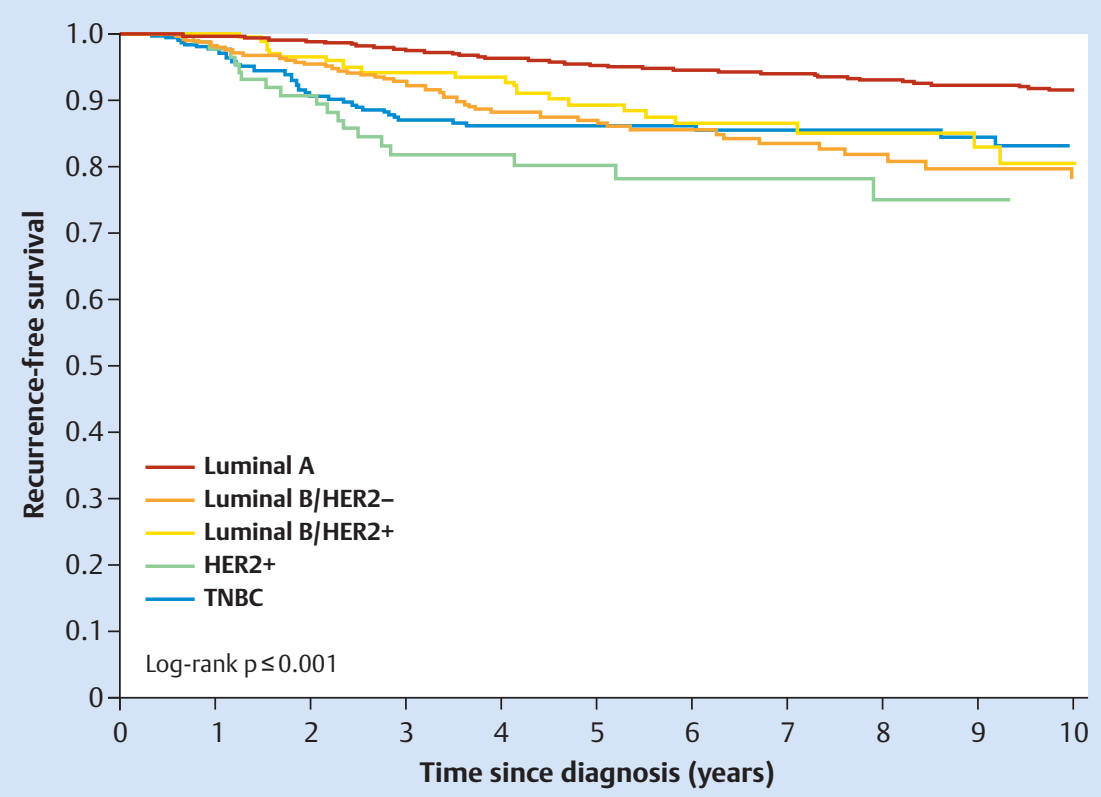

Fig. 2 Kaplan-Meier curve: survival without systemic recurrence categorized according to clinical subgroup.

Table 3 Risk factors for local, loco-regional and systemic recurrence (on multivariate analysis).

\begin{tabular}{|c|c|c|c|c|c|c|c|c|c|}
\hline \multirow[t]{2}{*}{ Characteristics } & \multicolumn{3}{|l|}{ LR } & \multicolumn{3}{|l|}{ RR } & \multicolumn{3}{|l|}{ DR } \\
\hline & HR & $95 \% \mathrm{Cl}$ & $\mathbf{p}$ & HR & $95 \% \mathrm{Cl}$ & $\mathbf{P}$ & HR & $95 \% \mathrm{Cl}$ & p \\
\hline \multicolumn{10}{|l|}{ Age (years) } \\
\hline$>\leq 50$ vs. $>50$ & 2.1 & $1.4-3.2$ & $<0.001$ & 1.1 & $0.5-2.5$ & 0.810 & 1.1 & $0.8-1.5$ & 0.404 \\
\hline \multicolumn{10}{|l|}{ PT stage } \\
\hline - T2 vs. $\mathrm{T} 1$ & 0.9 & $0.6-1.5$ & 0.805 & 0.5 & $0.2-1.1$ & 0.109 & 1.5 & $1.1-2.0$ & 0.007 \\
\hline - T3 vs. T1 & 4.1 & $1.8-9.3$ & $<0.001$ & 1.2 & $0.3-5.7$ & 0.821 & 2.3 & $1.4-4.0$ & 0.002 \\
\hline - T4 vs. $\mathrm{T} 1$ & 1.1 & $0.1-7.7$ & 0.962 & 2.1 & $0.5-8.7$ & 0.286 & 4.5 & $2.7-7.6$ & $<0.001$ \\
\hline \multicolumn{10}{|l|}{ pN stage } \\
\hline - N1 vs. N0 & 1.8 & $1.1-3.0$ & 0.017 & 3.5 & $1.5-8.1$ & 0.004 & 2.0 & $1.5-2.9$ & $<0.001$ \\
\hline - N2 vs. N0 & 1.7 & $0.8-3.9$ & 0.190 & 4.0 & $1.3-13$ & 0.018 & 2.9 & $1.9-4.3$ & $<0.001$ \\
\hline - N3 vs. N0 & 1.1 & $0.3-3.7$ & 0.898 & 10 & $3.5-28$ & $<0.001$ & 5.0 & $3.3-7.6$ & $<0.001$ \\
\hline \multicolumn{10}{|l|}{ Subtypes } \\
\hline - luminal B (HER2-) vs. luminal A & 2.4 & $1.4-4.1$ & 0.001 & 1.2 & $0.4-3.9$ & 0.704 & 2.0 & $1.4-2.8$ & $<0.001$ \\
\hline - luminal B (HER2+) vs. luminal A & 1.1 & $0.5-2.7$ & 0.753 & 2.2 & $0.6-7.9$ & 0.209 & 1.9 & $1.2-3.0$ & 0.007 \\
\hline HER2+vs. luminal A & 1.7 & $0.6-4.9$ & 0.297 & 2.6 & $0.6-12$ & 0.214 & 2.4 & $1.4-4.1$ & 0.001 \\
\hline - TNBC vs. luminal A & 2.4 & $1.4-4.2$ & 0.001 & 5.4 & $2.7-11$ & 0.001 & 2.2 & $1.5-3.2$ & 0.001 \\
\hline \multicolumn{10}{|l|}{ lymphovascular invasion } \\
\hline - yes vs. no & 1.3 & $0.7-2.5$ & 0.368 & 0.9 & $0.3-2.7$ & 0.916 & 1.4 & $1.0-2.0$ & 0.028 \\
\hline \multicolumn{10}{|l|}{ Margins } \\
\hline positive vs. negative & 0.6 & $0.2-1.6$ & 0.285 & * & * & * & 2.1 & $0.8-5.1$ & 0.121 \\
\hline \multicolumn{10}{|l|}{ multifocal/multicentric } \\
\hline yes vs. no & 1.1 & $0.6-1.9$ & 0.662 & 0.9 & $0.3-2.3$ & 0.790 & 1.2 & $0.9-1.6$ & 0.261 \\
\hline \multicolumn{10}{|l|}{ Type of surgery } \\
\hline - mastectomy vs. BCS & 0.4 & $0.2-0.7$ & 0.001 & 0.8 & $0.2-2.8$ & 0.748 & 1.4 & $1.0-1.9$ & 0.049 \\
\hline \multicolumn{10}{|l|}{ Systemic therapy } \\
\hline novs.yes & 1.5 & $0.8-2.8$ & 0.208 & 0.7 & $0.3-1.8$ & 0.479 & 2.2 & $1.4-3.5$ & 0.001 \\
\hline \multicolumn{10}{|l|}{ Radiation therapy } \\
\hline novs.yes & 4.3 & $2.6-7.2$ & $<0.001$ & 5.4 & $2.7-11$ & $<0.001$ & 1.4 & $1.0-1.9$ & 0.049 \\
\hline
\end{tabular}

LR: local recurrence; RR: loco-regional recurrence; DR: distant recurrence; BCS: breast-conserving surgery; HR: hazard ratio (adjusted); CI: confidence interval; * no statistical event

of LR but a significantly higher risk of DR; however, mastectomy had no recognizable effect on RR. Systemic therapy had a beneficial effect on preventing DR but did not affect LR and RR.
Comparison of recurrence rates during

the treatment period

To compare potential changes in recurrence rates we divided the observation period into 2 subperiods (1998-2005 and 20062011). No significant differences in 5-year LRFS, RRFS and DRFS were found between the 2 subperiods. 


\section{Discussion}

$\nabla$

This study evaluated and compared the risk factors for local recurrence, loco-regional recurrence distant recurrence in 3054 patients with a primary diagnosis of early-stage, non-metastatic breast cancer treated in HSK Wiesbaden hospital and EssenMitte hospital. The risk of recurrence was analyzed for different subgroups. In our total patient population, the rates of local recurrence and loco-regional recurrence at 5 years after primary diagnosis were very low with rates of 3.3 and $1 \%$, respectively. The rate of distant recurrence was $8 \%$. We showed that known risk factors such as age, nodal status, tumor size, lymphatic invasion in addition to undergoing systemic and radiation therapy affected recurrence rates in the different groups in varying ways. A continuous increase in the risk of LR, RR and DR (on multivariate analysis) was found for patients who had not undergone radiation therapy, with tumor stage $>\mathrm{T} 2$ or positive nodal status. Factors such as tumor-free margins and focality were not found to affect the risk of LR, RR and DR on multivariate analysis. The discussion about what constitutes the optimal margin for the prevention of local recurrence has been extremely controversial in recent years. After the initial requirement of a healthy resection margin of $5-10 \mathrm{~mm}$ in surgery for invasive carcinoma the current recommendation now proposes a healthy margin of $1 \mathrm{~mm}$ [28]. In their current recommendations, the Society of Surgical Oncology and the American Society for Radiation Oncology have stated that no ink on the tumor margin is sufficient as an adequate margin in invasive cancer $[30,31]$. This definition also aims to significantly reduce the rates of re-excision. In our patient population the rate of re-excision was $25 \%$ which is comparable to that given in other international publications [32,33], even though an accompanying ductal carcinoma in situ (DCIS) was present in $48 \%$ of our patients. Nevertheless, it can be reasonably assumed that the more restrictive definition of a tumor-free margin will lower the rate of re-excisions, particularly in view of the fact that approximately half of all re-excisions are done to extend an already existing healthy tumor-free margin $[32,33]$.

With regard to the risk of local recurrence for multifocal/multicentric $(\mathrm{MF} / \mathrm{MC})$ cancer, some of the data is controversial. In their study, Lynch et al. [34] reported no significant effect of focality on the risk of local recurrence compared to unifocal tumors and therefore concluded that breast-conserving therapy could be considered sufficiently safe in this patient cohort. However, in another study the authors showed that multicentricity but not multifocality was a negative prognostic factor with regard to the risk of systemic recurrence and overall breast-cancer-specific survival [35]. Other study groups [36,37] have reported significantly better survival for patients with unifocal tumors compared with multifocal/multicentric tumors. In our study multifocality/ multicentricity did not constitute a risk factor for the investigated types of recurrence. The current German recommendations stipulate performing mastectomy in patients with multicentric tumors [28].

In our study, age $\leq 50$ years was a risk factor for local recurrence (HR 2.1; 95\% CI: 1.4-3.2) but not for loco-regional or systemic recurrence. Earlier studies published in the literature reported the 5 -year LR risk for young women after BCS as ranging between 10 and 36\% [38-41]. In their evaluation of 17 prospective studies, the EBCTCG (Early Breast Cancer Trialists' Collaborative Group) showed that young women ( $<40$ years) after BCS had a significantly higher 10-year LR risk compared with older women ( $>70$ years) (36 vs. 9\%). This raises the question whether younger women with breast cancer should be advised against BCS. However, recent publications (and our own analysis) have reported a significantly lower 5-year LR risk of $5 \%$ in young women [20]. Laar et al. [42] elegantly demonstrated the improvement in the LR rate over the period 1988-2010 in women < 40 years of age. Although the mean 5-year LR rate averaged over the entire period was $7.5 \%$, it differed quite considerably across the different observation periods: for the period 1988-1998 it was $9.8 \%$; for 1999-2005 it was 5.9\%; for 2005-2010 it was 3.3\% ( $p=0.006)$. Because of the very low rates of recurrence in our patient population in the 2 observation periods, we found no significant differences between observation periods. Several studies which looked specifically at the safety of BCS in TNBC patients reported that these patients did not have an increased risk of LR if they underwent breast-conserving surgery $[43,44]$. In the current St. Gallen recommendations published for 2013, BCS is not considered absolutely contraindicated in patients < 35 years [29], nor do the first international consensus guidelines on the treatment of breast cancer in young women consider BCS to be contraindicated in young patients [45]. In our analysis, patients who had undergone mastectomy had a lower risk of LR; on the other hand they were found to be at higher risk of systemic recurrence. In our experience, patients with clearly advanced tumor stages and/or unfavorable tumor biology are more likely to have mastectomy procedures. However, surgery does not change the unfavorable tumor biology, meaning that while extensive surgery reduces the local risk of recurrence, it cannot change the risk of systemic recurrence.

Studies have shown that systemic therapy, whether it consists of anti-hormone therapy $[46,47]$, chemotherapy and/or anti-HER2 therapy [48], reduces the risk of recurrence. The findings in our study also demonstrated the impact of systemic therapy, in particular the impact of anti-HER2 therapy on patients with HER2positive cancer. These findings were in accord with those of previous studies $[49,50]$.

Patients with luminal-A tumors had excellent 5-year LRFS, RRFS and DRFS rates. In contrast, all patients with TNBC had a significantly higher risk of all forms of recurrence. 5-year DRFS was significantly lower in all subgroups compared to the luminal A tumor subgroup. In their meta-analysis of 12592 patients, Lowery et al. [51], showed that patients with TNBC or HER2+ subtypes had a significantly higher LR risk compared to patients with luminal/HER2- cancer, irrespective of the type of surgery (LR rate after BCS was $13.5 \%$; after mastectomy it was $12.9 \%$; mean follow-up: 57 months). We made the same observation in our study; however, the 5-year LR rate calculated in our analysis was significantly lower than in their study (TNBC and HER2+: 6\% respectively). Pilewskie et al. [52] investigated the effect of resection margins after BCS on the risk for LR and DR in 535 TNBC patients.

Our study has some limitations which must be mentioned. It was a retrospective analysis of two German breast centers. Neither the surgical procedures nor the other therapies (radiation therapy, chemotherapy/antibody therapy/hormone therapy) were standardized; instead all treatment was carried out to the best of the knowledge at the time and in accordance with existing national/international recommendations. The exclusion of patients who had neoadjuvant treatment could have distorted the patient population as it could have led to a cumulation of more favorable tumor biologies. The data on the pathological parameters (especially the determination of receptors) was not collected centrally. However, our study gives an unfiltered picture of the daily treat- 
ment regimens in these hospitals without bias or selection. Our study is an important contribution to healthcare research into the verification of clinical standards, a field that is becoming increasingly important. Moreover, to the best of our knowledge, our study constitutes the biggest analysis of German data on this issue to date.

\section{Conclusion}

\section{$\nabla$}

The LR and RR rates were very low in our patient population. The prognostic factors differed between investigated recurrence groups. Established risk factors such as age, nodal status and tumor size affected the different forms of recurrence to a varying extent. Based on the differentiation into subgroups, TNBC was associated with a significantly higher risk of local and loco-regional recurrence as well as a higher risk of systemic recurrence. The value of systemic therapy and radiation therapy was demonstrated by the significantly lower systemic recurrence rate in treated patients. In summary, the risk of recurrence must be evaluated on an individual basis and the treatment plan must consist of multimodal therapy which takes the individual tumor biology into consideration.

\section{Conflict of Interest \\ $\nabla$}

None.

\section{References}

1 Krebs in Deutschland. Häufigkeiten und Trends 2013. Online: www.rki. de

2 Online: www.onkozert.de

3 Del Turco MR, Ponti A, Bick U et al. Quality indicators in breast cancer care. Eur J Cancer 2010; 46: 2344-2356

4 Rutgers EJ. Quality control in the locoregional treatment of breast cancer. Eur J Cancer 2001; 37: 447-453

5 Arriagada $R$, Lê MG, Rochard $F$ et al. Conservative treatment versus mastectomy in early breast cancer: patterns of failure with 15 years of follow-up data. Institut Gustave-Roussy Breast Cancer Group. J Clin Oncol 1996; 14: 1558-1564

6 Fisher B, Anderson S, Bryant J et al. Twenty-year follow-up of a randomized trial comparing total mastectomy, lumpectomy, and lumpectomy plus irradiation for the treatment of invasive breast cancer. $\mathrm{N}$ Engl J Med 2002; 347: 1233-1241

7 Poggi MM, Danforth DN, Sciuto LC et al. Eighteen-year results in the treatment of early breast carcinoma with mastectomy versus breast conservation therapy: the National Cancer Institute Randomized Trial. Cancer 2003; 98: 697-702

8 van Dongen JA, Voogd AC, Fentiman IS et al. Long-term results of a randomized trial comparing breast-conserving therapy with mastectomy: European Organization for Research and Treatment of Cancer 10801 trial. J Natl Cancer Inst 2000; 92: 1143-1150

9 Veronesi U, Cascinelli N, Mariani L et al. Twenty-year follow-up of a randomized study comparing breast-conserving surgery with radical mastectomy for early breast cancer. N Engl J Med 2002; 347: 12271232

10 Blanchard DK, Donohue JH, Reynolds C et al. Relapse and morbidity in patients undergoing sentinel lymph node biopsy alone or with axillary dissection for breast cancer. Arch Surg 2003; 138: 482-487; discussion 487-488

11 Giuliano AE, Haigh PI, Brennan MB et al. Prospective observational study of sentinel lymphadenectomy without further axillary dissection in patients with sentinel node-negative breast cancer. J Clin Oncol 2000; 18: 2553-2559

12 Veronesi $U$, Paganelli $G$, Viale $G$ et al. A randomized comparison of sentinel-node biopsy with routine axillary dissection in breast cancer. $\mathrm{N}$ Engl J Med 2003; 349: 546-553
$13 \mathrm{Krag}$ DN, Anderson SJ, Julian TB et al. Sentinel-lymph-node resection compared with conventional axillary-lymph-node dissection in clinically node-negative patients with breast cancer: overall survival findings from the NSABP B-32 randomised phase 3 trial. Lancet Oncol 2010; 11: 927-933

14 Perou CM, Sorlie T, Eisen MB et al. Molecular portraits of human breast tumours. Nature 2000; 406: 747-752

15 Sorlie T, Perou CM, Tibshirani R et al. Gene expression patterns of breast carcinomas distinguish tumor subclasses with clinical implications. Proc Natl Acad Sci U S A 2001; 98: 10869-10874

16 Livasy CA, Karaca G, Nanda R et al. Phenotypic evaluation of the basallike subtype of invasive breast carcinoma. Mod Pathol 2006; 19: 264271

17 Jacquemier J, Ginestier C, Rougemont J et al. Protein expression profiling identifies subclasses of breast cancer and predicts prognosis. Cancer Res 2005; 65: 767-779

18 Dominici LS, Mittendorf EA, Wang X et al. Implications of constructed biologic subtype and its relationship to locoregional recurrence following mastectomy. Breast Cancer Res 2012; 14: R82

19 Tang P, Skinner KA, Hicks DG. Molecular classification of breast carcinomas by immunohistochemical analysis: are we ready? Diagn Mol Pathol 2009; 18: 125-132

20 Arvold ND, Taghian AG, Niemierko A et al. Age, breast cancer subtype approximation, and local recurrence after breast-conserving therapy. J Clin Oncol 2011; 29: 3885-3891

21 Millar EK, Graham PH, O'Toole SA et al. Prediction of local recurrence, distant metastases, and death after breast-conserving therapy in early-stage invasive breast cancer using a five-biomarker panel. J Clin Oncol 2009; 27: 4701-4708

22 Nguyen PL, Taghian AG, Katz MS et al. Breast cancer subtype approximated by estrogen receptor, progesterone receptor, and HER-2 is associated with local and distant recurrence after breast-conserving therapy. J Clin Oncol 2008; 26: 2373-2378

23 Voduc KD, Cheang MC, Tyldesley S et al. Breast cancer subtypes and the risk of local and regional relapse. J Clin Oncol 2010; 28: 1684-1691

24 Sinn P, Aulmann S, Wirtz R et al. Multigene assays for classification, prognosis, and prediction in breast cancer: a critical review on the background and clinical utility. Geburtsh Frauenheilk 2013; 73: 932940

25 Lux MP, Hildebrandt T, Bani M et al. Health economic evaluation of different decision aids for the individualised treatment of patients with breast cancer. Geburtsh Frauenheilk 2013; 73: 599-610

26 Empfehlungen gynäkologische Onkologie Kommission Mamma. Online: www.ago-online.de

27 NCCN Guidelines Version 3.2014. Online: www.nccn.org

28 Leitlinienreport der S3 Leitlinie für die Diagnostik, Therapie und Nachsorge des Mammakarzinoms. 2012. Online: http://www.krebsgesellschaft.de

29 Goldhirsch A, Winer EP, Coates AS et al. Personalizing the treatment of women with early breast cancer: highlights of the St Gallen International Expert Consensus on the Primary Therapy of Early Breast Cancer 2013. Ann Oncol 2013; 24: 2206-2223

30 Buchholz TA, Somerfield MR, Griggs JJ et al. Margins for breast-conserving surgery with whole-breast irradiation in stage I and II invasive breast cancer: American Society of Clinical Oncology endorsement of the Society of Surgical Oncology/American Society for Radiation Oncology consensus guideline. J Clin Oncol 2014; 32: 1502-1506

31 Moran MS, Schnitt SJ, Giuliano AE et al. Society of Surgical OncologyAmerican Society for Radiation Oncology consensus guideline on margins for breast-conserving surgery with whole-breast irradiation in stages I and II invasive breast cancer. J Clin Oncol 2014; 32: 1507-1515

32 Morrow M, Jagsi R, Alderman AK et al. Surgeon recommendations and receipt of mastectomy for treatment of breast cancer. JAMA 2009; 302: 1551-1556

33 McCahill LE, Single RM, Aiello Bowles EJ et al. Variability in reexcision following breast conservation surgery. JAMA 2012; 307: 467-475

34 Lynch SP, Lei X, Hsu L et al. Breast cancer multifocality and multicentricity and locoregional recurrence. Oncologist 2013; 18: 1167-1173

35 Lynch SP, Lei X, Chavez-Macgregor M et al. Multifocality and multicentricity in breast cancer and survival outcomes. Ann Oncol 2012; DOI: $10.1093 /$ annonc/mds136

36 Weissenbacher T, Zschage M, Janni W et al. Multicentric and multifocal versus unifocal breast cancer: is the tumor-node-metastasis classification justified? Breast Cancer Res Treat 2010; 122: 27-34 
37 Yerushalmi R, Kennecke H, Woods $R$ et al. Does multicentric/multifocal breast cancer differ from unifocal breast cancer? An analysis of survival and contralateral breast cancer incidence. Breast Cancer Res Treat 2009; 117: 365-370

38 Nixon AJ, Neuberg D, Hayes DF et al. Relationship of patient age to pathologic features of the tumor and prognosis for patients with stage I or II breast cancer. J Clin Oncol 1994; 12: 888-894

39 Kroman N, Holtveg H, Wohlfahrt J et al. Effect of breast-conserving therapy versus radical mastectomy on prognosis for young women with breast carcinoma. Cancer 2004; 100: 688-693

40 Bollet MA, Sigal-Zafrani B, Mazeau $V$ et al. Age remains the first prognostic factor for loco-regional breast cancer recurrence in young $(<40$ years) women treated with breast conserving surgery first. Radiother Oncol 2007; 82: 272-280

41 Cabioglu N, Hunt KK, Buchholz TA et al. Improving local control with breast-conserving therapy: a 27-year single-institution experience. Cancer 2005; 104: 20-29

42 van Laar C, van der Sangen MJ, Poortmans PM et al. Local recurrence following breast-conserving treatment in women aged 40 years or younger: trends in risk and the impact on prognosis in a population-based cohort of 1143 patients. Eur J Cancer 2013; 49: 3093-3101

43 Zumsteg ZS, Morrow M, Arnold B et al. Breast-conserving therapy achieves locoregional outcomes comparable to mastectomy in women with T1-2N0 triple-negative breast cancer. Ann Surg Oncol 2013; 20: 3469-3476

44 Adkins FC, Gonzalez-Angulo AM, Lei X et al. Triple-negative breast cancer is not a contraindication for breast conservation. Ann Surg Oncol 2011; 18: 3164-3173
45 Partridge AH, Pagani O, Abulkhair 0 et al. First international consensus guidelines for breast cancer in young women (BCY1). Breast 2014; 23 : 209-220

46 Fisher B, Bryant J, Dignam JJ et al. Tamoxifen, radiation therapy, or both for prevention of ipsilateral breast tumor recurrence after lumpectomy in women with invasive breast cancers of one centimeter or less. J Clin Oncol 2002; 20: 4141-4149

47 Davies C, Godwin J, Gray R et al. Relevance of breast cancer hormone receptors and other factors to the efficacy of adjuvant tamoxifen: patient-level meta-analysis of randomised trials. Lancet 2011; 378: 771-784

48 Kiess AP, McArthur HL, Mahoney K et al. Adjuvant trastuzumab reduces locoregional recurrence in women who receive breast-conservation therapy for lymph node-negative, human epidermal growth factor receptor 2-positive breast cancer. Cancer 2012; 118: 1982-1988

49 Sanpaolo P, Barbieri V, Genovesi D. Prognostic value of breast cancer subtypes on breast cancer specific survival, distant metastases and local relapse rates in conservatively managed early stage breast cancer: a retrospective clinical study. Eur J Surg Oncol 2011; 37: 876-882

50 Harris EE, Hwang WT, Lee EA et al. The impact of HER-2 status on local recurrence in women with stage I-II breast cancer treated with breastconserving therapy. Breast J 2006; 12: 431-436

51 Lowery AJ, Kell MR, Glynn RW et al. Locoregional recurrence after breast cancer surgery: a systematic review by receptor phenotype. Breast Cancer Res Treat 2012; 133: 831-841

52 Pilewskie $M$, Ho A, Orell $E$ et al. Effect of margin width on local recurrence in triple-negative breast cancer patients treated with breastconserving therapy. Ann Surg Oncol 2014; 21: 1209-1214 